\title{
HEAT FLUX DENSITY DISTRIBUTION ON THE ELECTRODES OF AN ARC*
}

\author{
BY \\ R. L. BISH \\ Materials Research Laboratory, Ascot Vale, Victoria, Australia
}

\begin{abstract}
A general formula is derived for the radiant heat flux density on the surfaces of the electrodes of an electric arc. The formula is applied to a vertical arc burning above a cone-shaped electrode. In the case of a $90^{\circ}$ cone, or flat plate, the heat flux density distribution shares the same general form as the Gaussian distribution. It is shown that the effect of decreasing the cone angle is to increase the flux density at the cone vertex and to diminish this flux elsewhere on the cone surface.
\end{abstract}

1. Introduction. Two major problems of arc welding are those of determining.

(i) the cooling rates in the body being welded, and

(ii) the melting rate of the rod.

Rosenthal [1] attempted to solve these problems by applying his moving heat source theory, but he assumed infinitely concentrated sources of heat. It seems clear, however, if we are to obtain accurate information concerning points close to the arc root, that we must take into account the distributed nature of the radiation from the arc; and indeed several writers have attempted to do this. Pavelic [2] and other writers [3] have, in fact, assumed that the distribution of heat flux density on a flat horizontal plate, due to a vertical arc, is Gaussian.

The true distribution of heat flux density on any shape of electrode may be derived from first principles, and it is the purpose of this paper to present the results of mathematical investigations of this matter. First the general theory will be developed, and then applied to particular cases.

2. General theory. Figure 1 shows an arc burning above the surface of an electrode of arbitrary shape. Let $V$ denote the potential at any point of the arc column, and $I$ the total current passing through the arc and around the external metallic circuit. The element of the arc column shown, and across which the potential drop equals $d V$, radiates power equal to $I d V$ watts through a solid angle equal to $4 \pi$ steradians. On the other hand the solid angle, $\omega$, subtended by a surface area, $S$, on the electrode is, by Gauss's formula [4],

$$
\omega=\int_{S} \mathbf{n} \cdot \nabla \frac{1}{r} d S,
$$

*Received September 13, 1988.

(C) 1989 Brown University 
where $\mathbf{n}$ is the unit normal vector at a point on $S$ and $r$ is the radial distance from the point of the arc column to this point. The power transmitted to $S$ from the arc element equals $\left(\frac{\omega}{4 \pi}\right) I d V$ so that, on integrating over the arc column, we obtain for the total power, $P$, radiated onto $S$

$$
P=\frac{I}{4 \pi} \int_{V_{1}}^{V_{2}} d V \int_{S} \mathbf{n} \cdot \nabla \frac{1}{r} d S
$$

where $V_{1}$ and $V_{2}$ are the potentials at the lower and upper ends, respectively, of the arc column. The variation of potential along a typical arc gap [5] is shown in Fig. 2.

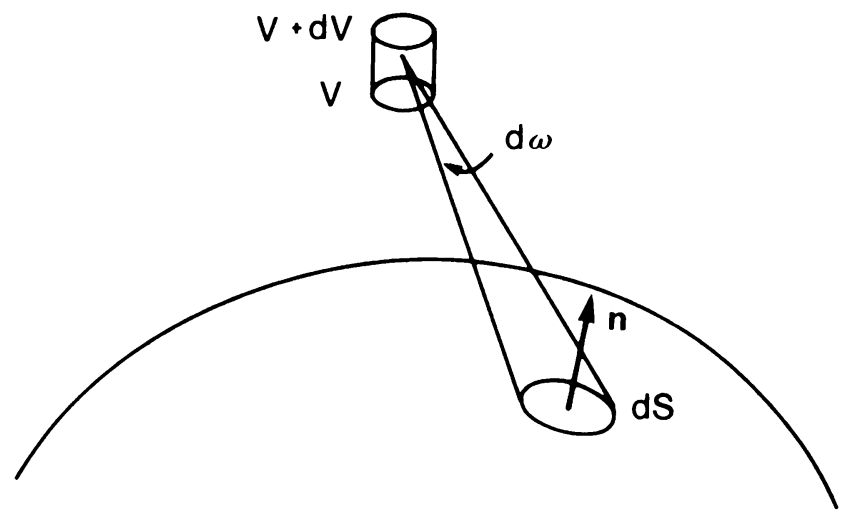

Fig. 1. Arc struck on an electrode of arbitrary surface geometry.

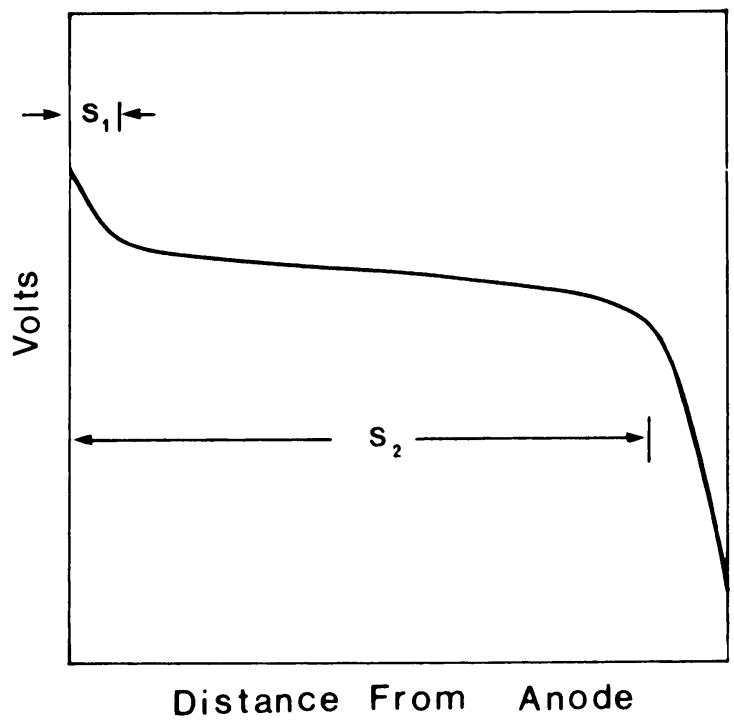

Fig. 2. Voltage variation along the axis of a typical high pressure arc. 
Over the central region collisions, ionizations, dissociations, and recombinations occur, causing this portion of the arc to radiate. At the gas/metal junctions ions (at the cathode), and electrons (at the anode), fall freely towards the electrode surfaces. Thus if we denote by $s$ distance along the arc, and assume $d V / d s$ equals a constant, $E$, over the radiating portion $s_{1}<s<s_{2}$, then, by Eq. (1), the normal heat flux density, $J$, equals

$$
J=\frac{E I}{4 \pi} \int_{s_{1}}^{s_{2}} \mathbf{n} \cdot \nabla \frac{1}{r} d s .
$$

We may now apply this formula to specific electrode geometries; in particular, the theory for a cone-shaped electrode proves most useful.

3. Cone electrodes. Figure 3 shows a midsection through a cone of semi-angle $\alpha$, with its axis vertical and collinear with the axis of a vertical arc.

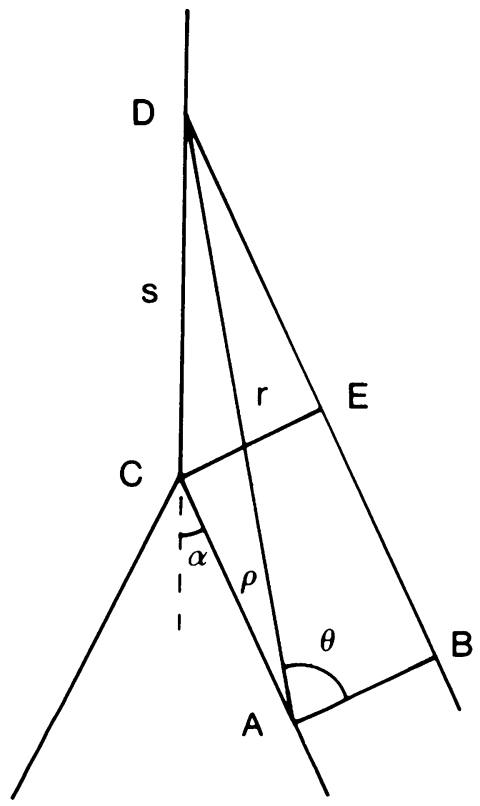

FIG. 3. Evaluation of the resolved component of $\operatorname{grad}(1 / r)$ normal to the cone surface CA. The arc axis is indicated by DC.

The position vector of a point, $\mathrm{A}$, on a cone surface relative to a point, $\mathrm{D}$, on the arc axis is $r$, and is distant $\rho$ from the cone vertex $C$. The length $C D$ has been resolved onto the normal to the cone surface, and has projected length $\mathrm{AB}$ or $\mathrm{CE}$, where D, E, and B are collinear and lie on a line parallel to CA. Now

$$
\mathbf{n} \cdot \nabla \frac{1}{r}=\mathbf{n} \cdot \mathbf{r} / r^{3}=\cos \theta / r^{2}
$$

where $r$ is the magnitude of DA and $\theta$ is the angle DAB. This equals $\mathrm{AB} / r^{3}$ or $\mathrm{CE} / r^{3}$ which equals $\mathrm{CD} \sin \alpha / r^{3}$. Writing $s$ for $\mathrm{CD}$, we also have, from the triangle $\mathrm{CDA}$,

$$
r^{2}=s^{2}+\rho^{2}+2 s \rho \cos \alpha
$$


so that $\mathbf{n} \cdot \nabla \frac{1}{r}=s \sin \alpha /\left(s^{2}+\rho^{2}+2 s \rho \cos \alpha\right)^{3 / 2}$. Thus, by Eq. (2),

$$
\begin{aligned}
J & =\frac{I E \sin \alpha}{4 \pi} \int_{s_{1}}^{s_{2}} \frac{s d s}{\left(s^{2}+\rho^{2}+2 s \rho \cos \alpha\right)^{3 / 2}} \\
& =\frac{E I}{4 \pi \rho \sin \alpha}\left(\frac{\left(\rho+s_{1} \cos \alpha\right)}{\left(\rho^{2}+s_{1}^{2}+2 \rho s_{1} \cos \alpha\right)^{1 / 2}}-\frac{\left(\rho+s_{2} \cos \alpha\right)}{\left(\rho^{2}+s_{2}^{2}+2 \rho s_{2} \cos \alpha\right)^{1 / 2}}\right) .
\end{aligned}
$$

Evaluating the limit as $\rho$ tends to zero, we find that

$$
\begin{aligned}
\lim _{\rho \rightarrow 0} J & =\frac{E I}{4 \pi \sin \alpha} \lim _{\rho \rightarrow 0}\left(\frac{1}{s_{1}}\left(1+\frac{s_{1}}{\rho} \cos \alpha\right)-\frac{1}{s_{2}}\left(1+\frac{s_{2}}{\rho} \cos \alpha\right)\right) \\
& =\frac{E I}{4 \pi \sin \alpha}\left(\frac{1}{s_{1}}-\frac{1}{s_{2}}\right) .
\end{aligned}
$$

4. Results. The arc used in metal/inert gas arc welding is typically millimetres in length while the anode fall may be as small as $0.01 \mathrm{~mm}$ [5]. Welding is generally carried out with the rod as the cathode.

For $s_{1}=0.1 \mathrm{~mm}$ and $s_{2}=5.0 \mathrm{~mm}$ the distributions given by Eqs. (3) and (4) for $\alpha=30^{\circ}$ and $90^{\circ}$ have been calculated and are shown plotted in Fig. 4. The case $\alpha=90^{\circ}$ corresponds, of course, to the flat plate.

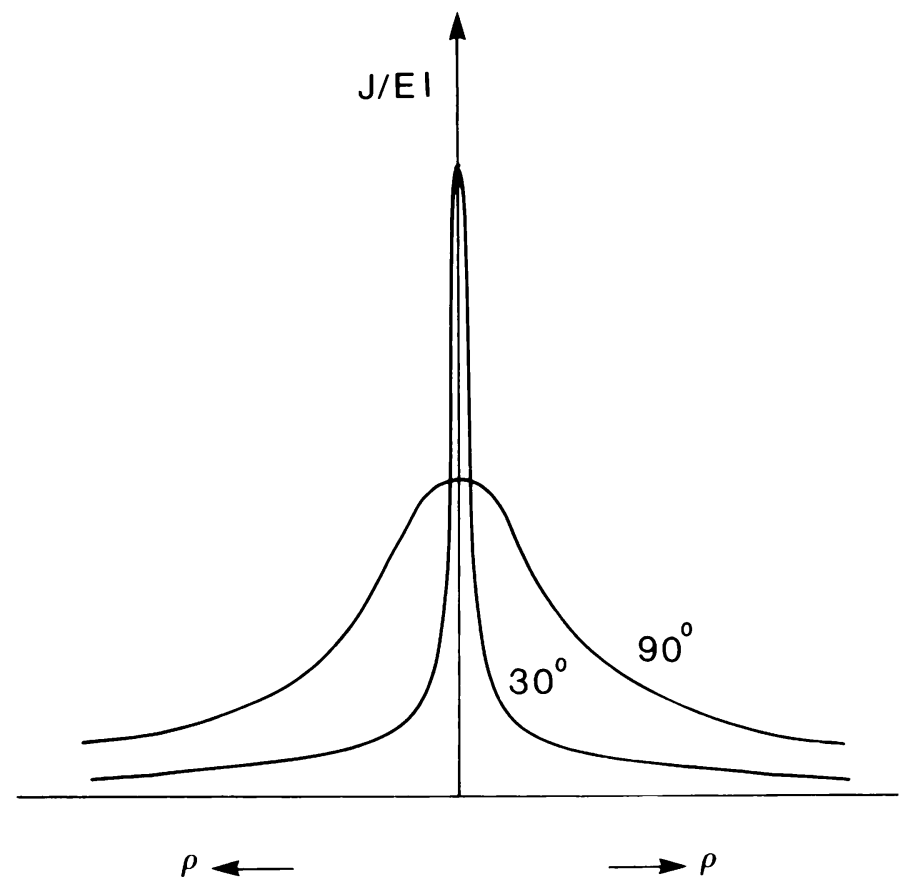

FIG. 4. Derived distributions of heat flux density on a cone of semiangle $30^{\circ}$, and on a flat plate. 
5. Discussion and conclusions. An assumption made in arriving at Eq. (3) is that no energy is absorbed by the shielding gas of the arc. This is not strictly true, of course, but it has been shown that in high pressure mercury vapour discharges the energy carried through the convection zone amounts to less than $1 \%$ of the total input [5]. A second assumption is that the arc column has no width. This seems reasonable since the column becomes highly contracted at the anode and cathode falls.

In the case of a flat plate, Eq. (4) shows that the flux density near the arc spot center is sensitive to the value of $s_{1}$. This means that, in welding using straight polarity, the electron mean free path must be accurately known before Eq. (3) can be expected to provide meaningful information for points near the arc spot center. Another quantity appearing in Eq. (3) that needs to be known is the axial field strength. This may be measured by one of several methods that Somerville describes [5]. Provided that these arc parameters can be accurately determined, Eqs. (3) and (4) should provide the necessary boundary values of the normal temperature gradient to be used in Rosenthal's moving heat source theory, enabling determinations to be made of the melting rate of the rod and the cooling rates in the plate.

\section{REFERENCES}

[1] D. Rosenthal, Mathematical theory of heat distribution during welding and cutting, Welding J. 20, $220(1941)$

[2] V. Pavelic, R. Tanbakuchi, O. A. Uyehara, and P. S. Myers, Experimental and computed temperature histories in gas tungsten-arc welding of thin plates, Welding J. 48, 295 (1969)

[3] H. A. Nied, Weld Pool Geometry Predictions Using a Two-Dimensional Heat Flow Model, Conference on Trends in Welding Research, Gatlinburg, Tennessee, 18-22 May 1986, edited by S. A. David, published by ASM, p. 21

[4] B. Spain, Vector Analysis, D. Van Nostrand, 1965, p. 85

[5] J. M. Somerville, The Electric Arc, Methuen, London, 1959, p. 22 